Ghana

External trade (1975) Imports: $\varnothing 909 \mathrm{mn}=\$ 788 \mathrm{mn}=£ 436 \mathrm{mn}$ Exports: $\varnothing 928 \mathrm{mn}=\$ 804 \mathrm{mn}=£ 445 \mathrm{mn}$

$\begin{array}{lclc}\text { Main imports } & \% \text { of total } & \text { Main exports } & \% \text { of total } \\ \text { Machinery } & 16 & \text { Cocoa } & 59 \\ \text { Chemicals } & 14 & \text { Timber } & 8 \\ \text { Crude oil } & 13 & \text { Aluminium } & 4 \\ \text { Food } & 12 & \text { Petroleum products } & 3 \\ \text { Motor vehicles } & 9 * & \text { Manganese ore } & 2 \\ \text { Textile yarns and fabrics } & 6 & & \\ \text { Main sources } & & \text { Main destinations } & \\ \text { United States } & 16 & \text { United Kingdom } & 15 \\ \text { United Kingdom } & 15 & \text { United States } & 11 \\ \text { West Germany } & 11 & \text { Netherlands } & 10 \\ \text { Nigeria } & 7 & \text { Switzerland } & 8 \\ \text { Japan } & 6 & \text { West Germany } & 8 \\ \text { Libya } & 5 & \text { Japan } & 7\end{array}$

\section{Special focus}

Cocoa prices (London, wholesale price)
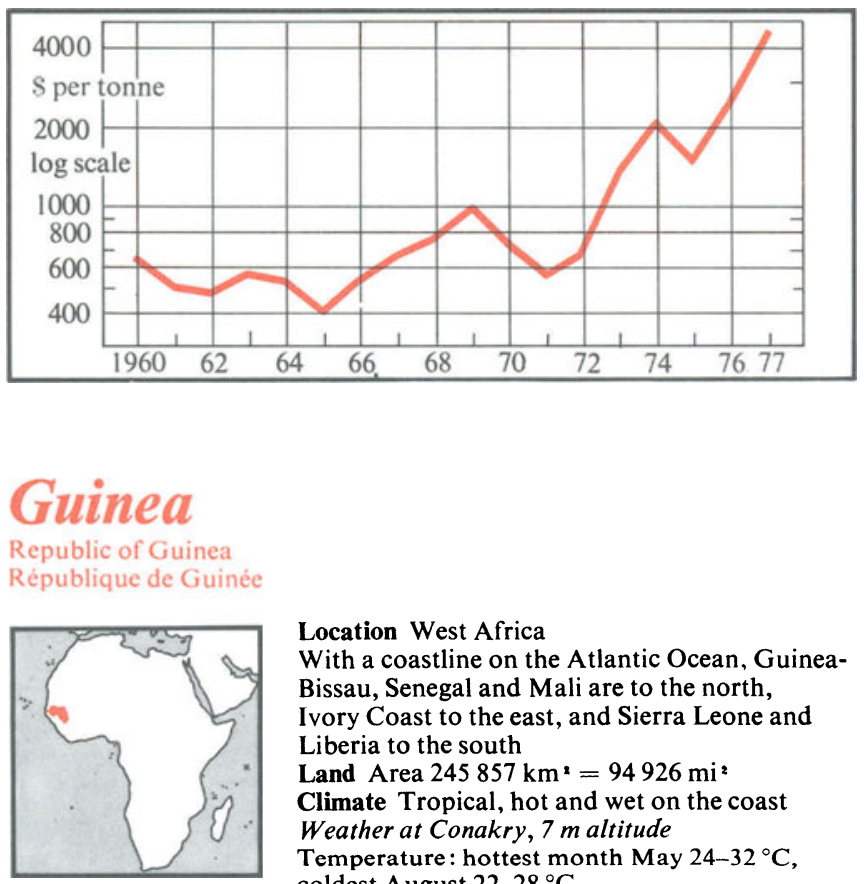

Location West Africa

With a coastline on the Atlantic Ocean, GuineaBissau, Senegal and Mali are to the north, Ivory Coast to the east, and Sierra Leone and Liberia to the south

Land Area $245857 \mathrm{~km}^{2}=94926 \mathrm{mi}^{2}$

Climate Tropical, hot and wet on the coast Weather at Conakry, 7 m altitude

Temperature : hottest month May $24-32{ }^{\circ} \mathrm{C}$, coldest August $22-28^{\circ} \mathrm{C}$

Rainfall (av monthly): driest month January $3 \mathrm{~mm}$, wettest July $1298 \mathrm{~mm}$ Time GMT

Measures Metric system

Monetary unit Syli (Sy) = 100 cauris; the Syli was introduced

October 1972, replacing the Guinean franc (G Fr) at Sy $1=$ G Fr 10.

Rate of exchange (1976 av) : par Sy $24.6853=$ SDR 1, free Sy $21.38=\$ 1$, Sy $38.62=£ 1$

\section{Summary}

Political One-party people's republic, which became independent October 2, 1958; formerly a French colony. Member of UN, OAU, Ecowas and an EEC ACP state

Economic A broadly based agricultural economy, with pineapples, coffee and palm kernels important export products; however, the main export earner is aluminium in the form of bauxite and alumina-accounting for some three-quarters of export earnings. Iron ore deposits near Liberia are being further developed

\section{People, resources and equipment}

Population (UN est) $19603.07 * * \mathrm{mn}, 19703.92 * * \mathrm{mn}, 19764.53 * * \mathrm{mn}$ Official Guinea population estimate $(1972): 5.14^{* *} \mathrm{mn}$, of whom $1.5^{* *} \mathrm{mn}$ living outside the country

Growth: 1960-70 2.5**\%pa, 1970-762.4**\%pa

Density (1976): $18^{* *}$ people per $\mathrm{km}^{2}$

Vital statistics (rate per 1000 people, $1970-75$ ): births $46.6^{*}$, deaths $22.9^{*}$
Cities (population in 000, 1972)

Conakry (capital) 526 Kindia $45^{\mathrm{a}}$ Labe $26^{\mathrm{a}}$ Siguiri $18^{\mathrm{a}}$ Kankan $176 \quad$ N'Zérékoré 26 $^{\mathrm{a}}$ Mamou $18^{\mathrm{a}}$

a 1967

Race (1976) Malinke 34** \%, Fulani 34** \%, Susu 10** \%, Kissi 6**\%, Kpelle 5**\%

Language French; local languages are also used, especially Malinke and

Susu

Religion (1976) Moslem 85**\%, Animist 14**\%, Christian 1**\%

Education (1971/72) Pupils 243 300*, teachers 7 800*

Labour force (1976) $2072000 *$; in agriculture $1700000 *(82 * \%)$

Personnel (1973) Physicians: 188, 1 per 22390 people

Standard of living

National income per person (1976): Sy $3300^{* *}=\$ 150^{* *}=£ 85^{* *}$

Consumption per person (1975): energy $92 \mathrm{~kg}$ coal equivalent,

electricity (production) $113^{*} \mathrm{~kW} \mathrm{~h}$, steel $4 \mathrm{~kg}$

Newspapers (1974): number 1 ; circulation 5000,1 per 1000 people

Telephones (Dec 1974): 10000,2 per 1000 people

Livestock $(000,1976)$ Cattle $1550^{*}$, sheep $420^{*}$, goats, $385^{*}$, pigs $35^{*}$

Hospital beds (1972) 6858,1 per 600 people

Roads (1975) $10000 * \mathrm{~km}=6000 * \mathrm{mi}$, density $0.04 * \mathrm{~km}$ per km*

Railways (1975) $902 \mathrm{~km}=560 \mathrm{mi}$, density $0.004 \mathrm{~km}$ per km²

A $1200 \mathrm{~km}$ line is planned to link Conakry with east Guinea iron ore mines near Liberia

Ships (registered, 1977) 11, total of 12597 gross tons

Ports Conakry, Kamsar

Airports Conakry, Kankan and 8 other airports with scheduled services

Durable equipment Radio sets (Dec 1974): 105000,24 per 1000 people

Passenger cars (Dec 1972): 10 200,2.5 per 1000 people, 1.0* per km of road

Commercial vehicles (Dec 1972): $10800,2.6$ per 1000 people,

$1.1^{*}$ per $\mathrm{km}$ of road

\section{Production, finance and trade}

\section{Gross domestic product}

1976 est: Sy $16000^{* *} \mathrm{mn}=\$ 750 * * \mathrm{mn}=£ 410^{* *} \mathrm{mn}$

Agricultural production index $(1970=100) 1976103$;

growth 1970-76 $0.4 \%$ pa

Main products (000 t) Agriculture (1976) Rice 375*, maize 320*,

cassava $480^{*}$, bananas $70^{*}$, plantains $215^{*}$, palm kernels $15^{*}$, palm oil $35^{*}$, coffee $5^{*}$, pineapples $15^{*}$, timber $\left(000 \mathrm{~m}^{3} 1975\right) 3139$. Other $(1975)$

Electricity (mn kW h) 500*, bauxite 7620 , alumina (1972) 700 , iron ore

(Fe content, 1970) 1040 , diamonds (000 CM) 80

Transport traffic Passenger-kilometres Rail (1967) $42 \mathrm{mn}$, air (1976) $26 \mathrm{mn}$

Cargo: tonne-kilometres Rail (1971) $341 \mathrm{mn}$, air (1976) $2.5 \mathrm{mn}$

Sea (1971) Goods loaded $1300000 * \mathrm{t}$, unloaded $550000 * \mathrm{t}$

Budget (1972/73; year ending Sept 30th)

Balanced at Sy $4500 \mathrm{mn}=\$ 210 \mathrm{mn}=£ 90 \mathrm{mn}$

External trade (1975) Imports: Sy $3800^{* *} \mathrm{mn}=\$ 180^{* *} \mathrm{mn}=£ 80^{* *} \mathrm{mn}$

Exports: Sy $3300 * * \mathrm{mn}=\$ 160 * * \mathrm{mn}=£ 70 * * \mathrm{mn}$

Main imports (1970) $\%$ of total Main exports (1971) $\%$ of total

Petroleum and products $14 \quad$ Alumina and bauxite

Textiles and clothing

Rice

14

10

Machinery and metals

Pineapples

Coffee

Main sources (1975)

France

Soviet Union

United States

Morocco

Belgium-Luxembourg

United Kingdom

Spain

$24^{*}$
$15^{*}$
$15^{*}$
$8^{*}$
$7^{*}$
$5^{*}$
$3^{*}$

Palm kernels

Bananas

72

10

Main destinations (1975)

United States

Spain

Soviet Union

Canada

West Germany

France

Jugoslavia

Special focus

Main non-agricultural products (000 tonnes)

Bauxite Alumina Iron ore (Fe content)

19601378

19651870

19661609

$1967 \quad 1639$

19682118

19692459

$1970 \quad 2490$

19712630

19722650

19733660

19747605

19757620

$\begin{array}{rr}185 & 388 \\ 522 & 378 \\ 525 & 305 \\ 530 & 356 \\ 531 & 935 \\ 572 & 1040 \\ 610 & 1040 \\ 665 & \text { na } \\ 700 & \text { na } \\ \text { na } & \text { na } \\ \text { na } & \text { na } \\ \text { na } & \text { na }\end{array}$

